\title{
LOS EFECTOS DE LAS ELECCIONES DE 1993 Y 1995 SOBRE EL SISTEMA DE PARTIDOS EN VENEZUELA
}

\author{
Marisa Ramos*
}

\section{INTRODUCCION}

Las elecciones presidenciales y legislativas celebradas en Venezuela en 1993 ponen de manifiesto un cambio sustancial en el régimen político que trasciende la importancia de una consulta electoral. La trascendencia de estas elecciones se refleja en una serie de cambios políticos en distintos niveles, el principal de los cuales es la transformación en el sistema de partidos existente desde 1958. Los datos que otorgan a estos comicios la trascendencia señalada son el descenso brusco en la participación electoral, la fragmentación del sistema de partidos, el aumento de la volatilidad electoral y el triunfo, por primera vez desde 1958, de un candidato presidencial respaldado por partidos que se sitúan al margen del bipartidismo tradicional de AD y COPEI, que habían constituido la "partiarquía" señalada por Coppedge (1994) como característica básica del sistema político venezolano.

En estas páginas se tratará de analizar los cambios que representan estas elecciones en relación a las características seculares del sistema de partidos venezolano, señalando los factores que los explican. Así mismo, se intentará llegar a alguna conclusión válida en cuanto al posible cambio del sistema de partidos, contrastando los resultados de estas elecciones con los primeros datos de las elecciones locales de diciembre de 1995. Se tratará, fundamentalmente, de analizar hasta que punto se puede seguir manteniendo la idea defendida por Mainwaring y Scully (1994: 17) según la cual Venezuela constituye un sistema de partidos institucionalizado. Esta caracterización puede llegar a ser un elemento importante si se asume que la existencia de un sistema de partidos institucionalizado es garantía tanto de estabilidad democrática como de una mayor gobernabilidad del sistema (1). Califican a un sistema de partidos como institucionalizado cuando cumple los siguientes requisitos: 1. Estabilidad en

(*) Doctora por el Instituto Universitario Ortega y Gasset. Actualmente, investigadora del Institute of Latin American Studies de la Universidad de Londres.

(1) Por gobernabilidad se podría entender la noción planteada por Alcántara (1995:1) entendida como "la situación en la que concurren un conjunto de condiciones favorables para la acción de gobierno que se sitúan en su entorno o que son intrínsecas a éste. El entorno a la acción de gobierno recoge aspectos ligados tanto al medio societal como al internacional. En el primero de ellos, los partidos políticos, como agentes vehiculadores de la representación popular, desempeñan un papel muy activo". las reglas y en la naturaleza de la competencia interpartidista; 2. Que los principales partidos tengan raíces estables en la sociedad; 3. Que los principales actores otorguen legitimidad al proceso electoral y a los partidos; y 4. Que la organización partidista sea autónoma e independiente tanto de sus líderes como de los movimientos u organizaciones que la crearon (Mainwaring y Scully, 1995: 4-5). En este sentido, la confirmación de la hipótesis de que Venezuela esté en un proceso de transformación que la llevaría a no ser considerada un sistema de partidos institucionalizado, podría constituirse en variable explicativa de la crisis de gobernabilidad que según algunos autores (García-Guadilla, 1995) sufre Venezuela.

\section{RESULTADOS ELECTORALES}

El día 3 de diciembre de 1993 los venezolanos disponían de dos papeletas para votar tanto al próximo presidente de la República como a los diputados y senadores que habrían de representarles en los siguientes cinco años. En las elecciones presidenciales resultó vencedor el ex-presidente Rafael Caldera, con un 30.46 por ciento de los votos, seguido de Claudio Fermín, de AD, con un 23.60 por ciento, Oswaldo Alvárez Paz, de COPEI con un 22.73 por ciento y Andrés Velásquez, de Causa R, con un 21.95 por ciento. Rafael Caldera se presentaba respaldado por un nuevo partido, Convergencia, que surgió como una ruptura de $\mathrm{CO}$ PEI, y por el MAS.

Conviene apuntar que para estas elecciones se estrena un nuevo sistema electoral para la Cámara de Diputados, así como para las asambleas legislativas de los estados. Se pasa de la representación proporcional en listas cerradas a un sistema de representación proporcional personalizada. El 50 por ciento de los diputados elegidos corresponden a circuitos uninominales. Esta reforma se produce impulsada por una opinión pública a favor de aumentar la personalización de la decisión electoral y forma parte del mismo ambiente que dio lugar a la elección directa de goberandores y alcaldes.

Según los primeros datos obtenidos de las elecciones locales celebradas el 3 de diciembre de 1995, AD consiguió el 
34.5 por ciento de los votos, COPEI el 21.2 por ciento, Causa R el 12.7 por ciento, el MAS el 10.8 por ciento y Convergencia el 8.7 por ciento (2).

La lectura de estos datos requieren una mirada tanto a las elecciones anteriores como a las características básicas del sistema político venezolano, marcado por una extrema centralidad política de los dos principales partidos políticos, $\mathrm{AD}$ y COPEI. Solo teniendo en cuenta las características anteriores pueden entenderse lo que, a mi juicio, son los dos principales datos a destacar, el ascenso de la abstención y el cambio que el mapa político resultante de estas elecciones otorga al sistema de partidos existente.

\section{Cuadro I \\ RESULTADOS DE LAS ELECCIONES LEGISLATIVAS DE 1988 Y 1993}

\begin{tabular}{lrrrrrr}
\hline & \multicolumn{3}{c}{ Elecciones 1988 } & \multicolumn{3}{c}{ Elecciones 1993 } \\
\multicolumn{1}{c}{ Partido } & \multicolumn{1}{c}{$\%$} & Diputados & Senadores & \multicolumn{1}{c}{$\%$} & Diputados & Senadores \\
\hline AD & 43.2 & 97 & 22 & 23.7 & 55 & 16 \\
COPEI & 31.0 & 67 & 20 & 22.7 & 53 & 14 \\
CAUSA R & 1.6 & 3 & - & 20.7 & 40 & 9 \\
CONVER. & - & - & - & 13.5 & 26 & 6 \\
MAS & 10.1 & 18 & 3 & 10.8 & 24 & 5 \\
OTROS & 13.9 & 16 & 1 & 8.5 & 5 & - \\
TOTAL & 100 & 201 & 46 & 100 & 203 & 50 \\
\hline
\end{tabular}

Fuente: Consejo Supremo Electoral

\section{LA PARTICIPACION ELECTORAL}

En cuanto al dato de la participación/abstención, Venezuela ha sido caracterizada siempre como uno de los países con un mayor índice de participación política en las elecciones. La participación en las distintas elecciones presidenciales y legislativas celebradas desde 1958 ha sido muy alta, siendo considerada comparativamente una de las más altas del mundo (3). El promedio de asistencia electoral de 1958 a 1988 ha sido de un 90.1 por ciento (Huneeus y Thibaut, 1993). Bien es verdad que este porcentaje es sobre la población inscrita. En tanto que el proceso de inscripción electoral no es automático, exigiendo una voluntad expresa del elector, el porcentaje real de participación debe medirse en relación al total de población en edad de votar. En este caso, el porcentaje de asistencia electoral desciende ligeramente, aunque sigue manteniéndose en niveles muy elevados en comparación con países con democracias consolidadas. Utilizando datos proporcionados por Rey (1989: $77 \mathrm{y}$ ss.) sobre la población electoral estimada por la Oficina Central de Estadística e Informática (OCEI) y los porcentajes de inscritos, se puede inferir un promedio de participación electoral sobre el total de la población con capacidad de votar del 84 por ciento (4). Molina (1988) establece dos

(2) Estas cifras corresponden a datos provisionales ofrecidos por el Consejo Supremo Electoral, Dirección de Estadísticas Electorales, aparecidos en El Universal, 15 de diciembre de 1995.

(3) Ver, por ejemplo, los datos que ofrecen Verba, Nie y Kim (1978).

(4) La participación en los diferentes comicios queda como sigue: $1958 \ldots 80.4 \%$ etapas en el desarrollo de la participación electoral. Sitúa la primera hasta 1973, en que la asistencia promedio fue un 93.4 por ciento y de 1973 a 1988 , con un 86.8 por ciento. La diferencia más significativa se produjo entre las elecciones de 1973 y las de 1978, con nueve puntos de diferencia.

\section{Cuadro II PARTICIPACIÓN ELECTORAL SOBRE LA POBLACIÓN INSCRITA ( por ciento)}

\begin{tabular}{|c|c|c|c|c|c|c|c|c|c|c|c|c|}
\hline Elecc. & 1958 & 1963 & 1968 & 1973 & 1978 & 1983 & 1984 & 1988 & 1989 & 1992 & 1993 & 1995 \\
\hline Legis. & 92.1 & 90.8 & 94.5 & 96.5 & 87.5 & 87.8 & & 81.7 & & & 60.2 & \\
\hline Pres. & 93.4 & 92.2 & 96.7 & 96.5 & 87.5 & 87.3 & & 81.9 & & & 58.3 & \\
\hline Local. & & & & & & & 59.3 & & 45.8 & 49.2 & & 46.11 \\
\hline
\end{tabular}

Fuente: Huneeus y Thibaut (1993) y Consejo Supremo Electoral

Sin embargo, teniendo en cuenta el minucioso análisis que realiza Molina (1988: 29-67), este alto nivel de participación tiene que ser matizado, analizando los factores del ordenamiento legal y del sistema de partidos que le afectan. Entre los que favorecen o estimulan la participación señala la obligatoriedad del voto (5), la movilización partidista unido al alto índice de militancia y la competitividad entre partidos, que otorga una alta probabilidad a la alternancia de partidos en el gobierno.

\section{Participación: elecciones legislativas} (1958-1993)

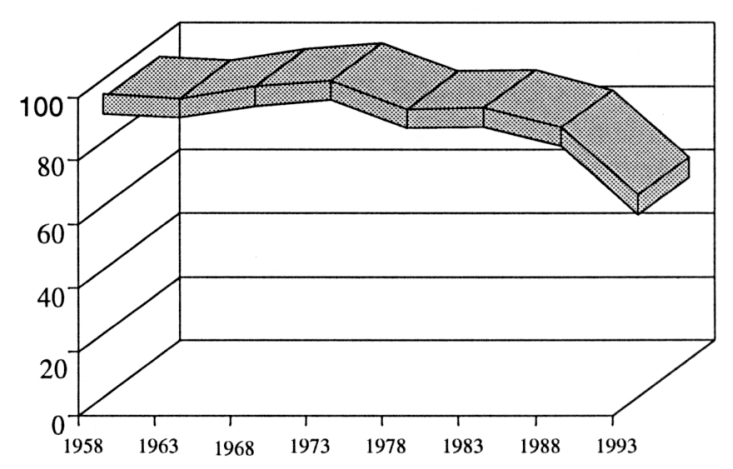

Así pues, sólo teniendo en cuenta los datos de participación en elecciones previas se puede comprender en su justa medida la importancia de un descenso en la participación de más de 20 puntos (82 por ciento en 1988 a 60.2 por ciento en 1993), cuando la mayor oscilación en las siete elecciones anteriores había sido de 8.8 puntos de diferencia (el menor índice de abstención se produjo en 1973, con un 3.5 por ciento, en tanto que el mayor porcentaje de abstención fue el 18 por
$1963 \ldots 75.1 \%$

$1968 \ldots 92.0 \%$

$1973 \ldots 89.5 \%$

$1978 * . .88 .0 \%$

$1988 \ldots 80.0 \%$

$1993 \ldots 58.3 \%$

* Según señala Rey (1989), los datos de población que calculó la OCEI para ese año fueron menores que la población realmente existente. Sin embargo, no proporciona más información sobre cuál es la fuente para llegar a esta conclusión. Si es así, lógicamente el porcentaje de participación es menor.

(5) Según el estudio de Baloyra y Martz (1979: 215) este factor se confirma como estimulador de la participación electoral, ya que en 1973, el 48.2 por ciento de la muestra señaló que no votaría si no fuera obligatorio. 
ciento de 1988). La lógica de la participación/abstención a nivel local es diferente a la lógica de las elecciones de carácter nacional, ya que generalmente tienen una menor participación. Si bien no se observan grandes oscilaciones en cuanto a los índices de abstención en las elecciones locales celebradas desde la entrada en vigor de la Ley Orgánica de Régimen Municipal, lo que se puede apuntar es la confirmación de la tendencia hacia la baja participación electoral, con índices que en ningún caso han superado el 50 por ciento. Esta cifra es aún más significativa si se tiene en cuenta que la elección directa de alcaldes y gobernadores fue un tema clave de presión entre la opinión pública a finales de la década de 1980.

Varias pueden ser las explicaciones de este descenso en la participación. Se pueden tomar las elecciones como indicador del apoyo social al régimen político existente. En este sentido, la participación electoral podría ser considerada como la forma en que los ciudadanos expresan su conformidad o disconformidad con el régimen existente. Siguiendo el análisis de Rey, se puede distinguir entre mecanismos normativos de adhesión al régimen y mecanismos utilitarios. Estos últimos se refieren a una "evaluación de tipo instrumental, en función de objetivos concretos que representan las preferencias o deseos efectivos de un actor y tiene que ver, por lo tanto, con el funcionamiento eficaz del sistema en relación a las aspiraciones o deseos efectos, concretos y personales de los evaluadores". Los mecanismos normativos se basan en una "evaluación en función de criterios imparciales, impersonales y objetivos y que por tanto no dependen de la capacidad del sistema para satisfacer, en algunos casos concretos, los deseos o intereses particulares del evaluador" (Rey, 1989: 191). Rey concluye que el régimen venezolano ha estado sustentado por mecanismos utilitarios, en los que los partidos políticos constituían la vía fundamental de manifestación. En tanto los partidos pierden su capacidad de satisfacer deseos efectivos de gran parte de los electores, la adhesión al régimen decrece.

Desde una perspectiva más institucional, teniendo en cuenta los factores que Molina (1988) señalaba como estimuladores de la alta participación electoral en Venezuela, hay que señalar que se han producido unos cambios sustanciales. En cuanto al factor de la obligatoriedad del voto, Molina y Pérez Baralt (1994: 85) apuntan que se ha perdido credibilidad en el voto obligatorio, por lo que esta pérdida puede haber actuado como desincentivo de la participación en tanto se ha eliminado toda sanción para quien no vota y se ha abandonado las campañas institucionales recordando esta obligatoriedad.

En cuanto al segundo factor señalado por Molina (1988), la competitividad entre los dos principales partidos, AD y COPEI cada vez se diferencian menos frente al electorado. En este sentido, a buen número de votantes les da exactamente igual $\mathrm{AD}$ que COPEI. Hay que recordar que a este acercamiento e identificación han contribuido los mismos partidos con un proceso de atenuación ideológica (6), tal como será analizado seguidamente.

(6) A este respecto es interesante utilizar los datos manejados en el proyecto "Elites parlamentarias en Amrica Latina" que dirige en la Universidad de Salamanca el profesor Manuel Alcántara, según los cuales la distancia en la autoubicación ideológica de los diputados venezolanos de AD y COPEI en la presente legislatura es de 0.10 en una escala de 0 (ninguna distancia) a 10 (máxima distancia). La distancia programatica media entre los diputados de estos partidos es de 0.06 en esta misma
Por último, Molina (1988) señalaba como uno de los factores estimulantes de la participación la movilización partidista. También en este caso se han producido cambios sustanciales en los últimos años. La crisis de los partidos y su pérdida de credibilidad ha generado una desconfianza hacia los mismos que anula incluso su alta capacidad de movilización. Se ha producido un debilitamiento de los lazos de identidad entre sociedad y partidos políticos tradicionales que ha redundado en una crisis de representación. A esta ha contribuido la crisis económica y el descenso de los partidos en su capacidad de control de recursos estatales, base de su alta capacidad de movilización social.

\section{FRAGMENTACION Y VOLATILIDAD DEL SISTEMA DE PARTIDOS}

Con las elecciones de diciembre de 1993 se rompe la tradición de bipartidismo atenuado existente en Venezuela desde 1973. Siguiendo a Molina y Pérez Baralt (1994), se puede decir que se pasa de un bipartidismo atenuado a un multipartidismo extremo. De 1973 a 1988, AD y COPEI han tenido una votación conjunta media de 76.8 por ciento en las elecciones legislativas y 90.1 por ciento en las presidenciales. En las elecciones de 1993 alcanzan únicamente el 45.9 por ciento y 45.3 por ciento respectivamente. En los comicios locales de diciembre de 1995 , AD y COPEI recuperan parte de su peso en el espectro político, al alcanzar entre los dos partidos una votación del 55.7 por ciento.

\section{Cuadro III}

\section{VOTACION CONJUNTA DE AD Y COPEI (por ciento)}

\begin{tabular}{lcc}
\hline Año & $\begin{array}{c}\text { Elecciones } \\
\text { presidenciales }\end{array}$ & Elecciones legislativas \\
\hline 1958 & 65.4 & 64.6 \\
1963 & 53.0 & 56.6 \\
1968 & 57.3 & 49.6 \\
1973 & 85.4 & 74.7 \\
1978 & 89.9 & 79.5 \\
1983 & 91.9 & 78.7 \\
1988 & 93.3 & 74.4 \\
1993 & 45.3 & 45.9 \\
\hline
\end{tabular}

Fuente: Estadísticas del Consejo Supremo Electoral

La fragmentación puede ser medida utilizando el indicador del número efectivo de partidos. Según el índice de Lakso y Taagapera (1979) (7), Venezuela ha tenido en el período 1973-1993 un número medio igual a 2.84 partidos, ascendiendo este número en las últimas elecciones presidenciales y legislativas a 5.4 partidos.

escala. Para calcular la distancia programática, Alcántara utiliza las variables de reducción del gasto público, aumento de los impuestos, la capacidad del Estado para resolver problemas, la fiabilidad de las elecciones, la consideración de la democraci como peligrosa y la posibilidad de que exista demócracia sin partidos políticos. Ver Alcántara (1995).

(7) Laakso y Taagapera miden cuantos partidos hay en un sistema de partidos ponderado por el tamaño de cada uno de ellos. Aplican la fórmula $\mathrm{Ns}=1 / \Sigma \mathrm{Pi}^{2}$, en la cual Ns es el número efectivo de partidos expresado en escaños y $\mathrm{Pi}$ es el reparto proporcional de escaños de cada partido. Ver Molina (1994), Mainwaring y Scully (1995) y Coppedge (1995). 


\section{NUMERO EFECTIVO DE PARTIDOS}

\begin{tabular}{lrrrrr}
\hline Elecciones & $\mathbf{1 9 7 3}$ & $\mathbf{1 9 7 8}$ & $\mathbf{1 9 8 3}$ & $\mathbf{1 9 8 8}$ & $\mathbf{1 9 9 3}$ \\
\hline Presidenciales & 2.72 & 2.52 & 2.41 & 2.27 & 5.22 \\
Legislativas & 3.36 & 3.10 & 2.97 & 3.37 & 5.59 \\
N $^{\mathbf{0}}$ Medio & 3.04 & 2.81 & 2.69 & 2.82 & 5.40 \\
\hline
\end{tabular}

Fuente: Molina Vega y Pérez Baralt (1994: 68).

Esta fragmentación se manifiesta con la entrada en escena de dos partidos diferentes a los existentes hasta ese momento, a costa de la pérdida de apoyo a los que se consideraban centrales en la vida política venezolana, AD y COPEI. El MAS, tercer partido en las elecciones de 1988, sigue manteniendo un caudal de votos similar al de entonces. Por tanto, lo realmente novedoso es la irrupción en escena de CAUSA R y el surgimiento de Convergencia. Cada uno de ellos se explica de forma distinta.

La irrupción de Causa $\mathrm{R}$ está claramente influida por el proceso de descentralización llevado a cabo desde 1989. Venezuela ha sido un país fuertemente centralizado, aunque nominalmente la Constitución de 1961 señalaba que constituía un sistema federal. Sin embargo, los gobernadores de los estados eran nombrados por la presidencia de la República, y los alcaldes tampoco eran electos. Además, hasta 1984, las elecciones locales se celebraban en el mismo acto que las presidenciales y legislativas, con lo cual el arrastre que ejercían estas últimas sobre aquellas era evidente. En 1989 se aprobó la Ley Orgánica de Régimen Municipal, la Ley de Descentralización Regional y la Ley de Elección de Gobernadores, después de varios años de presiones sociales, lideradas entre otros actores sociales y políticos, por el movimiento vecinal. Las primeras elecciones que se celebraron tras este proceso descentralizador, en 1989, ya pusieron de manifiesto los importantes cambios que se estaban gestando a nivel de la identificación partidista y que se reflejaron en la fuerza que adquirieron agrupaciones independientes y grupos de electores que concurrieron en solitario o en coalición con los partidos políticos tradicionales. En 1992, estas pautas se confirmaron, dando lugar a un cambio básico de mentalidad política, en tanto se comprobó que era posible un mapa político sin la omnipresencia de $\mathrm{AD}$ y COPEI. Este dato explica de manera evidente el triunfo de Causa R. Es impensable que un partido que apenas consiguió un 1.65 por ciento en las elecciones de 1988 , consiga un 22 por ciento en las siguientes si no hubiera sido por la proyección nacional que los triunfos en las elecciones locales les reportó, sobre todo, el triunfo de Andrés Velásquez, su líder, en la gobernación del Estado Bolívar en las elecciones de 1989 y 1992 y, con un carácter más simbólico, el triunfo en la Alcaldía de Caracas del candidato causerrista, Aristóbulo Istúriz, en diciembre de 1992. Los resultados obtenidos por Causa $\mathrm{R}$ en las elecciones locales de 1995 suponen, sin embargo, una pérdida considerable del caudal de votos obtenidos en las elecciones de 1993, no consiguiendo revalidar el triunfo en ninguno de estos espacios y no alcanzando prácticamente ninguna gobernación ni alcaldía importante. Con estos resultados se confirmaría la hipótesis de que el fuerte apoyo conseguido por Causa $\mathrm{R}$ en las elecciones de 1993 fue producto del arrastre que la pro- yección de triunfos locales dio a esta formación política, apoyo que debe interpretarse más como vía de canalización de la protesta y el desencanto hacia los partidos tradicionales que como respaldo al programa propio de Causa $\mathrm{R}$.

El surgimiento de Convergencia se relaciona con la característica del fuerte presidencialismo venezolano, que arrastra en buena medida el resto de votaciones. Como explicación del surgimiento de Convergencia, hay que tener en cuenta los graves acontecimientos que conoció Venezuela en el año 1992, con los dos intentos de golpe de Estado del 4 de febrero y del 29 de noviembre. Estos intentos golpistas son la expresión máxima de la grave crisis institucional que sufrió el país y que se manifiestan en el fuerte apoyo popular que recibió la primera intentona y en especial su cabecilla, Hugo Chávez. Estos golpes pusieron de manifiesto una fuerte crisis de liderazgo político. En este contexto, el ex-presidente Rafael Caldera adquirió un protagonismo notable, realizando unas declaraciones en un punto de equilibrio difícil entre la justificación de la intentona y el apoyo al régimen democrático. Su voz disonante en esta coyuntura crítica, unido a su ya reconocido respeto y prestigio, le fue lanzando con fuerza a la arena política. Sin embargo, la negativa de la dirección copeyana a presentarle como candidato a la presidencia, a pesar del claro apoyo popular que suscitaba, le condujo a abandonar el partido que el mismo había fundado y a llevarse tras de sí a un nutrido grupo de copeyanos. Convergencia, por tanto, ha sido una agrupación electoral formada para arropar a un candidato. Al hilo de esto hay que decir que Caldera recibió además el apoyo en su candidatura presidencial del MAS. Nuevamente, los datos de las elecciones locales de 1995 confirman esta hipótesis teniendo en cuenta el drástico descenso del apoyo a Convergencia sin Rafael Caldera en la arena electoral, 8.6 por ciento.

El índice de volatilidad (8) es también un ejemplo de los cambios sustanciales que está experimentando el sistema de partidos. Siguiendo lo señalado por Mainwaring y Scully (1995), Venezuela ha tenido un índice de volatilidad, de 18.8 en las legislativas en el período comprendido entre 1973 y 1993, estando por debajo de la media latinoamericana y por encima de la europea. La volatilidad de la última elección con respecto a la de 1988 asciende al 34.48 por ciento.

\begin{tabular}{ccccc}
\multicolumn{1}{c}{ Cuadro V } \\
INDICE DE VOLATILIDAD SOBRE \\
ELECCIONES LEGISLATIVAS ( por ciento) \\
\hline $\mathbf{1 9 7 3}$ & $\mathbf{1 9 7 8}$ & $\mathbf{1 9 8 3}$ & $\mathbf{1 9 8 8}$ & $\mathbf{1 9 9 3}$ \\
30.0 & 10.44 & 11.73 & 8.48 & 34.48 \\
\hline
\end{tabular}

Elaboración propia sobre datos del Consejo Supremo Electoral

Siguiendo a Coppedge (1995), dos son los modelos de análisis de los cambios en los índices de volatilidad, el modelo de identificación (modelo Michigan) y el modelo económico. Según el primero, el comportamiento electoral se

(8) Este índice resulta de la suma de las diferencias netas en porcentaje de votos ganados o perdidos por cada partido de una elección a otra, y luego dividido por dos. El cálculo se hizo únicamente teniendo en cuenta las elecciones legislativas desde 1973 a 1993. 
explicaría, básicamente, por factores de lealtad partidista, en tanto que según el modelo económico, los votantes variarían de preferencias partidistas de acuerdo a cálculos económicos. Parece asumido que en el comportamiento electoral intervienen tanto factores de un tipo como de otro (Coppedge, 1995), pero puede considerarse la existencia de un predominio de un tipo de factores sobre otros. Según los análisis que él realiza sobre el conjunto latinoamericano, parecen más decisivos los factores de identificación que los de carácter económico. De acuerdo a esta idea, en Venezuela el índice relativamente bajo de volatilidad en el período de 1973 a 1988 podría explicarse por la fuerte identificación partidista en torno a los dos principales partidos. A esta fuerte identificación habría contribuido, según Coppedge (1995), la fuerte organización partidista.

La centralidad de los partidos políticos en general como actores fundamentales del modelo sociopolítico venezolano es una característica unánimemente aceptada. La constitución de $\mathrm{AD}$ y COPEI como partidos modernos de masas (9) y el control que han tenido del aparato estatal, ha generado un sistema que Coppedge (1993) ha denominado como partiarquía. La capacidad de representación de sectores sociales amplios fue decisiva para incorporar masivamente a la población a un sistema político de cualquier otra forma débil. Durante las primeras décadas del régimen democrático, los conflictos sociales se canalizaron por la vía partidista utilizando como recurso la enorme renta petrolera y, cuando no fue suficiente, el endeudamiento público. Este hecho produjo una capacidad de representación de intereses muy elevada, dotando al sistema de amplias dosis de legitimidad así como una relativa eficacia teniendo en cuenta el objetivo principal propuesto por la clase política, cual era el mantenimiento del régimen y la relativa paz social. Así, la estabilidad del sistema se aseguró durante las dos primeras décadas con relativa facilidad.

AD y COPEI han marcado la vida política desde 1958 y se han alternado en el poder desde entonces hasta las últimas elecciones de 1993. La literatura referida a la política venezolana no pone en duda la característica de la fuerte presencia de estos dos partidos en la vida política y social del país y su papel como mediadores fundamentales en la relación Estado-sociedad (10). Así, por ejemplo, ya Levine (1973: 8) (11)señalaba que "básicamente la política venezolana puede ser descrita como un sistema de partidos. Los vehículos básicos de acción política son los partidos, quienes monopolizan la acción política". Ahora bien, tal como muestra Coppedge (1993), generalmente esta fortaleza se había visto como una característica positiva ya que se consideraba responsable, sobre todo por el acierto de sus líderes, de haber conseguido guiar la democracia y conducir sus respectivos partidos para frenar los conflictos y mantener un régimen democrático estable. Sin embargo, desde visio-

(9) En el sentido weberiano de ser resultado de la implantación del sufragio universal para incorporar a la política a nuevos sectores sociales (masas) que habían estado marginados de la misma. Por tanto, se contrapone a los partidos de notables o constituidos en torno a la figura de un caudillo. Para realizar esta función, requieren una organización, una ideología y un programa político.

(10) Ver, entre otros, Vaivads (1992) (1966)

11) Ver también Blank (1973), Ray (1969), Baloyra y Martz (1979) y Martz nes más críticas y con posterioridad a la década de 1970, cuando la crisis política ya era evidente, se ha distinguido entre el ideal democrático y el régimen basado en el bipartidismo AD-COPEI, haciendo a este último blanco de las críticas. Esta postura crítica con respecto a los partidos políticos se ha extendido entre los especialistas del sistema político venezolano, hasta el punto que se ha colocado como punto central de reforma la revisión del sistema de partidos políticos (12).

Entre las razones que explican la extrema centralidad de los dos principales partidos en la vida política venezolana, asumiendo un papel incluyente de la mayoría de la sociedad (Vaivads, 1992: 61), como partido moderno de masas, posiblemente haya sido una de las más importantes la inexistencia de un sistema organizacional extenso (Levine y Kornblith, 1995). Se puede establecer que es esta debilidad, entendida como la escasa capacidad para organizarse y articular intereses, la que dió lugar a que los partidos políticos se desarrollaran y se fortalecieran extraordinariamente, cooptando, asimilando y controlando cualquier intento posterior en este sentido. Se plantea que el "vacío organizacional" que existía cuando surgieron los partidos políticos fue el que impulsó su fortaleza (Levine, 1973) (13). Si bien es cierto que cuando surgieron los partidos políticos principales no existían organizaciones fuertes, que los partidos crearon sindicatos y federaciones campesinas sin ninguna intención de que fueran autónomas y que las organizaciones que lucharon contra la dictadura se aliaron a los partidos políticos como actores que lideraban esa lucha, (Coppedge, 1993: 152), no se puede obviar el hecho de que con posterioridad a 1958 , los partidos políticos han tenido un papel activo en la cooptación y control de cualquier forma de organización diferenciada y autónoma. En este sentido, se pueden presentar como indicadores el hecho de que desde los años 60, los dirigentes de las organizaciones sociales son electos en comicios que siguen líneas partidistas. Este es el procedimiento que se sigue en las elecciones sindicales, desde el nivel de las fábricas hasta la confederación nacional; lo mismo ocurre con las organizaciones campesinas, en el Colegio de Abogados, en el Colegio de Médicos, arquitectos y empleados públicos y también en las organizaciones de gobierno estudiantil (Coppedge, 1993: 152).

Uno de los indicadores más significativos de la fortaleza de los partidos políticos y de su importancia como instituciones mediadoras entre la sociedad y el Estado es el nivel de militancia partidista. A través de una encuesta realizada por la Universidad del Zulia, el 23.5 por ciento de la población declaró, en 1989, estar afiliada a algún partido político,

(12) Coppedge (1993: 12) señala que Venezuela sufre de un grado extremo de partiarquía, un síndrome que significa que los canales de representación están bloqueados, tanto al margen de los partidos como al interior de ellos. Señala que, comparativamente, "many other countries manifest some of these symptoms to some degree, but none of them suffers as severely from as many as Venezuela does". Como estudio significativo del grado de crítica hacia los partidos político y la necesidad de su reforma interna, ver COPRE (1991).

(13) Literalmente, Levine (1973: 28), señala; "in a situation resembling an organizational vacuum, AD was the only force to fill the gap". En esta misma línea, Pinto (1991: 119) apunta que "el partido político ha mostrado una singular capacidad para compatibilizar y articular aspiraciones y posiciones que inicialmente mostraban una irreductible apariencia (...). Esta (capacidad articuladora) encuentra su anclaje en el accidentado proceso en el cual el partido político se constituyó en la única institución mediadora capaz de elaborar y presentar discursivamente, con la coherencia requerida, un proyecto de organización de la sociedad". 
porcentaje relativamente alto $\mathrm{y}$, en cierta forma, incongruente si se tiene en cuenta que en otra pregunta sobre la opinión acerca de los partidos políticos, el 76.5 por ciento declaró tener una opinión desfavorable sobre los partidos políticos (Pardo, 1992: 243). Del cruce de ambas cuestiones, se deduce que un alto porcentaje de afiliados mantiene una actitud crítica con respecto a los partidos políticos.

Esta fortaleza y centralidad se ha manifestado, fundamentalmente, a través de la relación simbiótica entre estos partidos y el Estado y de la debilidad organizativa de la sociedad de forma autónoma. En cuanto al primer punto, su papel como fundadores del régimen, el papel protagonista que adquirieron en el proceso, el carácter consociacional de la relación entre ellos y la debilidad de otros actores, junto con la eficacia en el tratamiento a actores en principio que podrían ser competetivos (14), produce una asimilación temprana y progresiva del aparato estatal, lo que les ha otorgado el papel protagonista en la relación sociedadEstado.

Por otro lado, la relación de estos partidos con la sociedad obliga a tratar la calidad de la representación de los mismos. Ya se ha señalado la posible caracterización de AD y COPEI como partidos de masas en el sentido de que bajo su paraguas se acogen sectores sociales muy diversos, tanto en cuanto a diferencias de procedencia económica y social como a una no muy marcada división ideológica. Este punto fue variando progresivamente desde los primeros tiempos de desarrollo partidista, en que la matriz ideológica estaba muy marcada, a las últimas décadas, en que se limaron los extremos ideológicos fuertes. Las diferencias ideológicas entre ambos partidos (15), aunque siempre presentes, se fueron suavizando con el transcurso de la experiencia democrática. La absoluta prioridad otorgada a la cuestión electoral, junto a la preservación y consolidación de unas maquinarias organizativas cada vez más penetrantes en la sociedad, ansiosas por el aumento de su militancia, debilitaron la definición ideológica de AD y COPEI (Combellas, 1991). En este proceso de pragmatización, pasaron a ser lo que se llama un partido "atrapatodo" (catch-all-party). Este hecho se hizo manifiesto en los programas ofertados, sin ninguna línea ideológica clara, confirmando la frase de Downs (1957) (16) según la cual los partidos elaboran programas para ganar las elecciones, en lugar de ganar las elecciones para implementar sus programas.

En resumen, se podría decir que la capacidad de representación social que tuvieron los dos principales partidos durante años ha sido alta, pero basada en elementos no tanto ideológicos, sino de carácter clientelista y utilitarios. A este

(14) El caso de los militares es el más claro, a los que se trató con un especial favor, tanto política como económicamente.

(15) En un primer momento, se consolidaron en torno a dos ideologías, socialdemocracia y socialcristianismo (Combellas, 1991). A pesar de las lógicas diferencias ideológicas, compartieron dos ideas básicas: la instauración del sistema de democracia representativa y el principio básico del intervencionismo estatal en lo social y en lo económico.

(16) El caso de AD y COPEI confirmaría la teoría de Downs (1957) según la cual, en un sistema bipartidista, los partidos deben por un lado conservar a sus votantes $y$, por el otro, ganar a los llamados indecisos, independientes o los que prefieren votar por los minoritarios. Para captar a este sector del electorado, se atenúa la presentación de los extremos ideológicos, con el fin de no entrar en colisión con las ideas de los sectores cuyo voto quiere captarse. En este proceso, ambos partidos terminarán pareciéndose cada vez más entre sí y ubicándose en el centro del espectro ideológico. respecto, conviene enfatizar la característica de la identificación partidista como el tipo de identificación básica del venezolano durante varias décadas. Hay que tener en cuenta la característica ya señalada por Baloyra y Martz (1979: 192-193) en el sentido de que, en Venezuela, la identificación partidista, en términos de independiente, apolítico o partidista, era la que más determinaba las actitudes políticas, más que cualquier otro factor, como pudieran ser la ideología, el status socioeconómico, el contexto comunitario, el género o la edad. Sin embargo, lo que conviene tener presente es que aunque han primado los factores de identificación partidista sobre cualquier otro, en la base de esta fuerte identificación partidista, por las condiciones del desarrollo político venezolano, se encuentran criterios utilitarios (o de base económica) de adhesión a los principales partidos políticos.

\section{EFECTOS EN EL SISTEMA DE PARTIDOS}

Se lanzaba en la introducción la hipótesis de que Venezuela estuviera iniciando un proceso que la conduciría a dejar de pertenecer al grupo de países con un sistema de partidos institucionalizado, según la calificación de Mainwaring y Scully (1995). Para llegar a confirmar tal idea se han tomado en cuenta los cambios que afectan al primer y cuarto de los requisitos que señalan estos autores como imprescindibles para poder hablar de tal institucionalización: estabilidad en las reglas y la naturaleza de la competencia interpartidista y la autonomía de la organización partidista tanto con respecto a sus líderes como a los movimientos que los crearon. Utilizando para medir el primer criterio el índice de volatilidad, se comprueba que aunque Venezuela constituye uno de los casos con un índice de volatilidad media que está por debajo del promedio latinoamericano, si se desagrega este número medio sale a la luz la profunda transformación que en este aspecto han significado las elecciones de diciembre de 1993.

En cuanto al cuarto requisito, es evidente que el tiempo actúa como factor estabilizador. Las últimas elecciones han supuesto la entrada en la arena política de dos partidos sumamente dependientes de su líder, en el caso de Convergencia, hasta el punto de albergar dudas razonables acerca de su continuidad sin la figura de Rafael Caldera, o dependientes del contexto político que favoreció su ascenso electoral, en el caso de Causa R). Es decir, existen dos partidos con poca autonomía, rasgo que confirmarían los resultados de las elecciones locales de 1995.

El criterio de la fragmentación partidista es de más difícil evaluación, ya que no parece aceptable vincular el número de partidos con la estabilidad democrática, tema que ya ha sido suficientemente tratado en la ciencia política. Sin embargo, lo que si parece indudable es que la fragmentación partidista da lugar a un nuevo sistema de partidos, surgimiento que Lipset y Rokkan (1967) ligan a la existencia de coyunturas críticas históricas.

Por último, hay que señalar, así mismo, cambios en lo que se refiere al tercer criterio señalado por Mainwaring y Scully (1995) referente a la legitimidad 
que los actores políticos otorgan al proceso electoral y a los partidos políticos. En este sentido, el aumento del índice de abstención puede interpretarse como un descenso en la legitimidad que los electores otorgan al proceso electoral.

Sin embargo, a la vista de los datos de diversos estudios de opinión pública, de ello no puede derivarse una deslegitimación del sistema democrático. Resulta interesante comparar los datos que ellos ofrecen con otros conseguidos en fechas posteriores, en que la crisis sociopolítica ya era evidente. El apoyo social de la población al mecanismo de las elecciones en 1973 era de un 67 por ciento de los entrevistados, en tanto que un 73 por ciento se oponía a cualquier tipo de golpe militar. Al mismo tiempo, un 45 por ciento expresaron ser críticos con respecto al régimen democrático (Baloyra y Martz, 1979). Torres (1991), en un estudio realizado en 1987, muestra que el 23 por ciento de los entrevistados manifestó estar contento con el régimen democrático, un 62.9 por ciento más o menos y un 13.9 por ciento pensaba que debía ser sustituido. Ahora bien, teniendo en cuenta este último porcentaje, un 22 por ciento pensó que debería ser sustituido por una mejor democracia, de lo que deduce Torres (1991) que el porcentaje de la población antisistema está, tan solo, en torno a un 10 por ciento. No se observan diferencias importantes con respecto a los datos ofrecidos por una encuesta similar realizada diez años antes (29.5 por ciento; 55.5 por ciento y 15.1 por ciento respectivamente para las tres preguntas). En la encuesta que maneja Myers (1993), realizada en mayo de 1992, tras el primer intento de golpe de Estado, se comprueba que el respaldo al sistema democrático ha continuado siendo alto, superior al 80 por ciento, aunque de éstos, el 40 por ciento manifestó preferir un sistema democrático pero sin los actuales partidos políticos. Solo un 8 por ciento de la población declaró preferir un gobierno militar fuerte. Las encuestas realizadas con posterioridad al segundo intento confirma esta tendencia, ya que solo el 9 por ciento de los entrevistados declaró estar a favor del golpe militar (Templeton, 1995).

En suma, lo que estos datos ponen de manifiesto es que el sistema democrático ha sido y sigue siendo reconocido como el mejor sistema posible para la mayoría de la población, habiéndose deslegitimado, en cambio, algunas de sus principales instituciones, en especial, los partidos políticos. Sin embargo, conviene recordar la idea de Linz (1987) de que la pérdida de apoyo de todos los actores políticos en un régimen democrático puede llevar muy fácilmente a una erosión de legitimidad, proceso que puede haberse iniciado en los últimos años.

En este sentido, podría interpretarse el resultado de las elecciones de 1993 como expresión del rechazo hacia los partidos políticos tradicionales en tanto principales instancias de deslegitimación. Se podría rescatar como elemento positivo el hecho de que la protesta y el descontento se canalizaran democráticamente a través de la vía electoral. Sin embargo, una vez expresado este rechazo en un momento crítico, las maquinarias partidistas tradicionales han comenzado a recuperarse de acuerdo a los resul- tados de las elecciones locales de 1995. Por tanto, no se podría concluir que el sistema de partidos venezolano haya variado sustancialmente.

\section{BIBLIOGRAFIA}

AlCÁNTARA, MANUEl (1995) "Gobernabilidad y elite política en América Latina”, mímeo.

BALOYRA, EnRIQUe y MARTZ, John D. (1979) Political Attitudes in Venezuela. Societal Cleavages and Political Opinion, University of Texas Press, Austin y Londres.

BLANK, DAVID E. (1991) "El reto ideológico de los partidos políticos venezolanos" en MAGALLANES, Manuel V. (Dir.) Liderazgo e Ideología, Publicaciones del Consejo Supremo Electoral, Colección del Cincuentenario, $n^{\circ} 11$, Caracas.

Comisión Presidencial para la Reforma del Estado (COPRE) (1991) Venezuela, democracia y futuro. Los partidos políticos en la década de los 90 . Ed. Copre, Caracas.

COPPEDGE, MIchael (1993) "Partidocracia y reforma en una perspectiva comparada" en SERBIN, ANDRÉS, STAMBOUli, ANDRÉS, MCCOY, JENNIFER Y SMITH, WILLIAM (Eds.) Venezuela: la democracia bajo presión, Ed. INVESP-Nueva Sociedad, Caracas.

Coppedge, Michael (1994) Strong Parties and Lame Ducks, Stanford University Press, Stanford, California.

COPPEDGE, Michael (1995) "Freezing in the Tropics: Explaining Party System Volatility in Latin America", mímeo.

DOWNS, ANTHONY (1957) An economic theory of democra$c y$, Harper, New York.

Huneeus, Carlos Y Thibaut, Bernhard (1993) "Venezuela" en NOHLEN, DiETER (Coord.) Enciclopedia Electoral Latinoamericana y del Caribe, Instituto Interamericano de Derechos Humanos, San José, Costa Rica.

LAAKSO, MARCKU Y TAAGAPERA, REIN (1979) "The Effective Number of Parties: A Measure with Application to Western Europe", en Comparative Political Studies, vol. 12, n. ${ }^{\circ}$, abril, pp. 3-27.

LeVine, Daniel H. (1973) Conflict and Political Change in Venezuela, Princeton University Press.

LeVine, Daniel H. Y KoRnblith, MiRiam (1995) "Venezuela: The Life and Times of the Party System" en MAINWARING, SCOTT Y SCUlly, Timothy R. (Eds.) Building Democratic Institutions. Party Systems in Latin America, Stanford University Press, Stanford, California.

MaINWARING, SCOTT Y SCUlly, TimOTHY (1995) "Party Systems in Latin America" en MAINWARING, SCOTT Y SCUlly, Timothy R. (Eds.) Building Democratic Institutions. Party Systems in Latin America, Stanford University Press, Stanford, California.

MARTZ, JOHN D. (1966) Acción Democrática: Evolution of a Modern Political Party in Venezuela, Princeton University Press, Princeton, New Jersey.

MOLINA, JoSÉ ENRIQUE (1988) "La participación electoral en Venezuela" en Cuestiones Políticas, n' 4 , Universidad del Zulia. 
MolinA, José ENRIQUE y PÉREZ BARALT, CARMEN (1994) "Venezuela: ¿un nuevo sistema de partidos? en Cuestiones Políticas, $\mathrm{n}^{\circ} 13$, Universidad del Zulia.

MYERS, DAVID J. (1993) "Percepciones de una democracia bajo presión ¿decadencia inevitable o refundación? en SERBIN, ANDRÉs, STAMBOUli, ANDRÉs, MCCOY, JENNIFER Y SMITH, WILliAm (Eds.) Venezuela: la democracia bajo presión, Ed. INVESP-Nueva Sociedad, Caracas.

Molina, José EnRIQUE, Pereira, VAlia y VAIVAdS, HENRY (1995) “¿Puede superar la democracia venezolana su crisis? El papel de los partidos políticos” en Perelli, Carina, Picado, Sonia y Zovatto, DANIEL (Comps.) Partidos y clase política en América Latina, Ed. IIDH-CAPEL, Costa Rica.

PARDO, HERNÁN (1992) "Juventud e identidades políticas" en Magallanes, Manuel V. (Dir.) La esencia de la democracia. Partidos políticos y crisis, Publicaciones del Consejo Supremo Electoral, Colección del Cincuentenario, $n^{\circ} 13$, Caracas.
PinTO, LeONCIO (1992) "El partido político y la estabilidad de la democracia en Venezuela" en Cuestiones Políticas, $\mathrm{n}^{\circ} 7$, Universidad del Zulia.

RAY, TALTON F. (1969) The Politics of the barrios of Venezuela, University of California Press, Berkeley \& Los Angeles.

REY, JUAN CARLOS (1989) El futuro de la democracia en Venezuela, Serie Estudios, Colección Idea, Caracas.

TEMPLETON, ANDREW (1995) "The evolution of Popular Opinion" en GoOdMAN, LOUIS W., MENDELSON, JohanNa, NAIM, MoISÉS, TulChin, JOSEPH S., Y BLAND, GARY (eds.), Lessons of the Venezuelan Experience, The Woodrow Wilson Center Press y The Johns Hopkins Univeristy Press.

VAIVADS, HENRY (1992) "Partidos políticos y crisis" en MAgAllanes, MANUEl V. (Dir.) La esencia de la democracia. Partidos políticos y crisis, Publicaciones del Consejo Supremo Electoral, Colección del Cincuentenario

\section{RESUMEN}

La autora analiza los cambios ocurridos en las dos últimas convocatorias electorales en Venezuela. Sus observaciones muestran cómo un sistema de partidos tradicionalmente fuerte, bipartidista, se recompone a partir de la crisis política derivada de la dificultades económicas que vive este país. Emergencia de nuevas fuerzas, altas tasas de volatilidad y de desidentificación política son algunos de los nuevos procesos que aparecen en la vida política venezolana.

\section{ABSTRACT}

The author analyzes the changes which have happened in the last two elections in Venezuela. Her observations show how a strong traditionally party system, bipartidist, it's recomposed from the political crisis provoked by economic problems in the country. The emergency of new political forces, high rates of volatility and political desafection are some of the new processes which appeared in the Venezuelan political life.

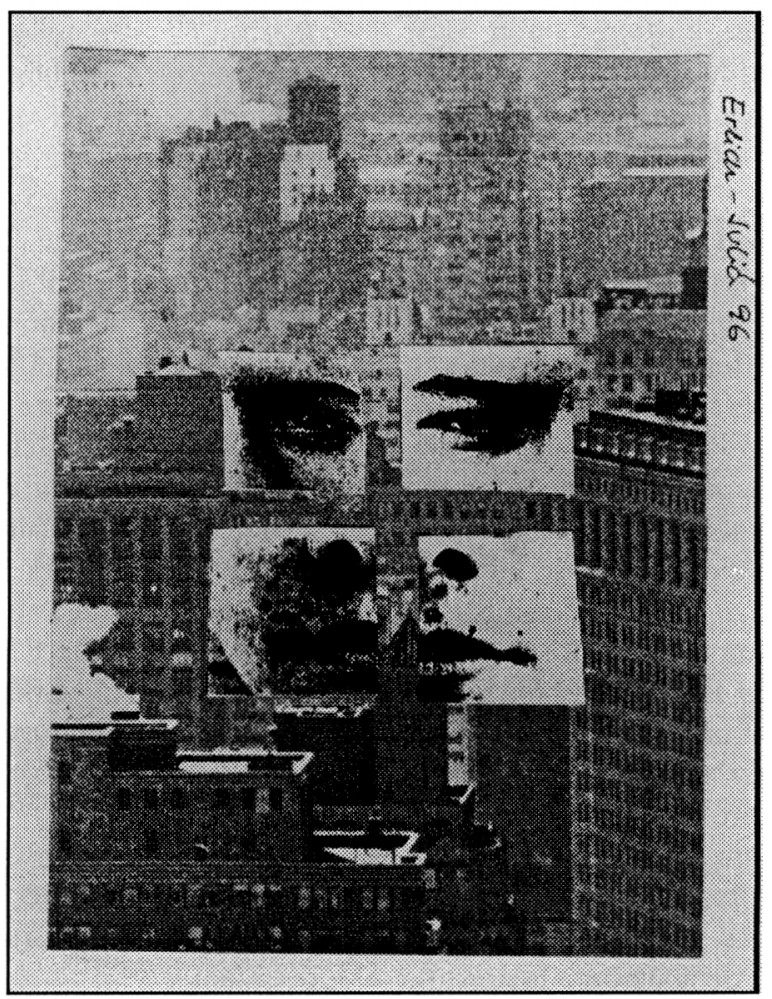

